\title{
Local plastic strain inhomogeneity in a ZEK100 Mg alloy
}

\author{
C.W. Sinclair ${ }^{1}$ and G. Martin ${ }^{1,2}$ \\ ${ }^{1}$ Department of Materials Engineering, The University of British Columbia, Vancouver, Canada V6T $1 Z 4$ \\ 2 SIMaP - Grenoble University, BP. 46, 101 rue de la Physique, 38402 Saint Martin d'Hères, France
}

\begin{abstract}
Magnesium alloys have great potential for lightweighting applications in the transportation industry. It is known, however, that $\mathrm{Mg}$ and $\mathrm{Mg}$-alloys exhibit large plastic strain heterogeneity. Such heterogeneity is important both from the perspective of formability and fracture if such sheet materials are to be stamped. Also, such observations are important for our fundamental understanding of how plasticity spreads in such materials. In this work we have sought to couple together local plastic strain measurements using digital image correlation with full-field crystal plasticity simulations with the idea of identifying and quantifying the plastic strain heterogeneity. A recently developed ZEK-100 alloy has been studied, as this alloy has shown promise as a wrought sheet product.
\end{abstract}

\section{EXPERIMENTAL METHODOLOGY}

E-beam lithorgraphy (Fig. 1) has been used to generate a regular grid on the surface of a ZEK-100 tensile specimen cut from a thermomechanically processed sheet material having a fully recrystallized microstructure and an average grain size of $\sim 30 \mu \mathrm{m}$ (see (Martin, 2013), (Martin, 2012) for a more detailed description of the e-beam lithorgraphy and image correlation technique). The size of the grid $(5 \mu \mathrm{m} \times 5 \mu \mathrm{m})$ was small enough to ensure that several grid points were located within each grain.

Prior to mechanical testing EBSD was performed on the area containing the lithographically developed grid (Fig. 2). The microstructure is seen to be well recrystallized and the texture is that typically of the ZEK alloys, i.e. a weak split basal texture with intensity spread along the transverse direction of the sheet. This texture is important considering the expected mechanical response. Because most grains are oriented with their caxis perpendicular to the rolling/tensile direction it is not anticipated that extension twinning will play a significant role in deformation (a fact that was confirmed after deformation by EBSD measurements).

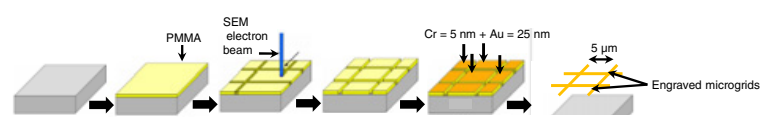

(a)

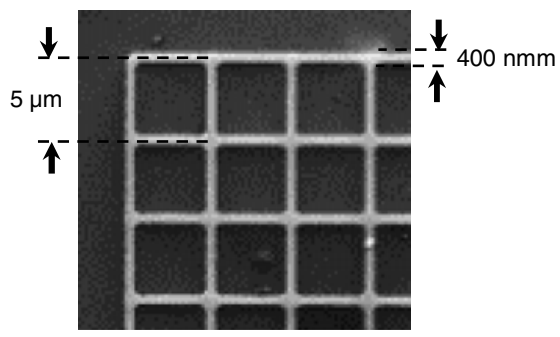

(b)

Figure 1. Schematic description of the e-beam lithography process for making the regular grid shown in (b). The grid intersection points were used for digital image correlation.

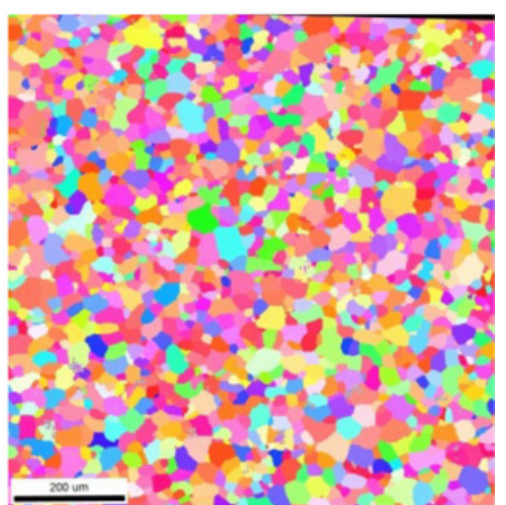

(a)

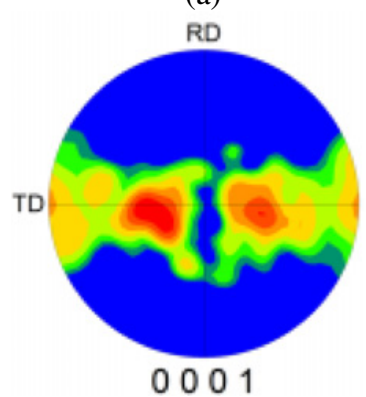

(b)

Figure 2. a) EBSD inverse pole figure map (colours corresponding to orientations parallel to ND) measured in the area containing the grid impsed by e-beam lithography $b$ ) $<0001>$ pole figure from the same EBSD map showing the TDsplit basal texture characteristic of this Mg-rare earth alloy.

\section{STRAIN HETEROGENEITY}

Figure 3 shows the macroscopic tensile stress-strain curve for the alloy as well as pure $\mathrm{Mg}$ tested parallel to the prior rolling direction. Other tests were done on the alloy to where the same sample was deformed to plastic strains of approximately $2 \%, 4 \%, 7 \%, 10 \%$ and $14 \%$. After each increment of strain the sample was unloaded and image correlation was performed using CorrelManuV developed by Bornet et al. (Bornert, 2010). Figure 4 shows the distribution of plastic strain found in the 


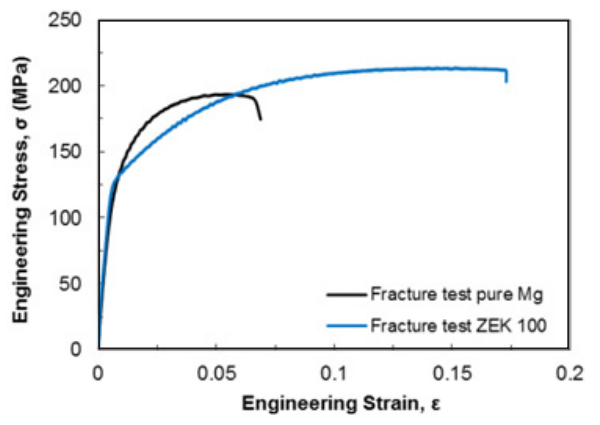

Figure 3. Tensile tests performed on the ZEK100 alloy as well as on pure Mg showing the improved ducitility of the ZEK alloy.

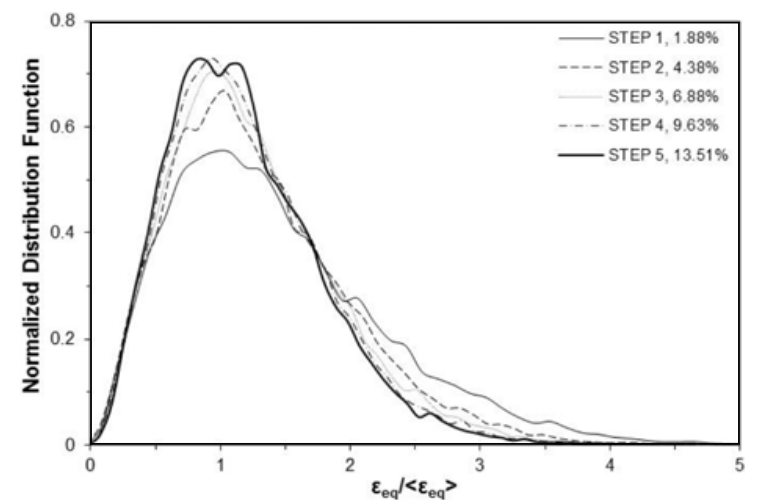

Figure 4. Histogram of local plastic strain (Von Mises equivalent strain) measured by image correlation after different levels of macroscopically imposed plastic strain. material. It is seen that the width of this plastic strain distribution is significantly larger than that found for FCC materials (Rollett 2010) and that the regions of large strain localization are particularly located close to grain boundaries. Using full-field VPFFT crystal plasticity simulations (Lebensohn 2001) it has been shown to be possible to reproduce these features if a sufficiently large CRSS anisotropy is chosen between basal and non-basal slip. This leads to large differences in the stress required to achieve deformation in adjacent grains leading to strain localization within 'weaker' grains that are well oriented for soft basal slip.

In summary, large intragranular plastic strain heterogeneity is found in the magnesium alloy ZEK100. This plastic strain heterogeneity appears to be linked to the anisotropy of CRSS on basal versus non-basal slip systems under the conditions of testing where slip is the dominant deformation mechanism.

\section{References}

G. Martin, C. W. Sinclair, J.-H. Schmitt, Scripta Materialia. 68 (2013) 695-698.

G. Martin et al., Experimental Mechanics 2012.

M. Bornert, F. Vales, H. Gharbi, D.N. Minh, Strain 2010;46:33.

R. A. Lebensohn, Acta Materialia 2001;49.

A. D. Rollett et al., Modelling and Simulation in Materials Science and Engineering 2010;18. 